\title{
Usefulness of the Electrocardiogram as a Tool to Localize Culprit Vessel Occlusion Site in Acute ST - Segment Elevation Myocardial Infarction
}

\author{
Mohamed Abdelhady Shama ${ }^{1 *}$, Mohamed Naseem Hussaien², \\ Mona Adel ELsaiedy ${ }^{2}$ and Raghda Ghonimy Elsheikh ${ }^{2}$ \\ ${ }^{1}$ Department of Emergency Medicine and Traumatology, \\ Tanta University Faculty of Medicine, Egypt \\ ${ }^{2}$ Department of Cardiovascular Medicine, Tanta University Faculty of Medicine, Egypt \\ *Corresponding author
}

\section{Keywords}

Electrocardiogram,

Acute ST -

segment,

Myocardial

infarction,

Coronary

artery

Article Info

Accepted:

15 May 2019

Available Online:

10 June 2019

A B S T R A C T

The objective of this study was to assess diagnostic accuracy of the ECG localization of culprit vessel occlusion site and compare it with coronary angiographic findings. This study analyzed 180 cases that came with acute myocardial infarction to cardiology department of Tanta University Hospital in a period from the first of May 2016 up to the end of May 2018. Typical chest pain lasting more than 30 minutes accompanied by ST-elevation at the J-point in two contiguous leads associated with elevation of cardiac markers were included in this study but we exclude patients with Previous history of myocardial infarction, Previous history of coronary artery bypass graft (CABG) surgery, ECG evidence of left bundle branch block (LBBB), preexcitation, paced rhythm and acute coronary syndrome other than STEMI. Among all myocardial infarction patients, ECG shows left anterior descending (LAD) branch affection in $116 / 180$ patients (64.44\%), which was as follow; $13.33 \%$ proximal to S1, $6.67 \%$ proximal to D1 and $44.44 \%$ distal to D1. Right coronary artery (RCA) presented in $56 / 180$ patients $(31.11 \%$ ) as follow; $11.11 \%$ proximal to RV branch and $20 \%$ distal to RV branch. Left circumflex artery (LCX) is represented only in $8 / 180$ patients $(4.44 \%)$. ECG is an easily and widely available inexpensive tool which helps in the routine clinical practice to categorize and optimize STEMI patients by detection of artery occluded into either right coronary artery or left anterior descending coronary artery or left circumflex coronary artery as the infarcted related culprit vessel and predict the possible complication 


\section{Introduction}

Acute coronary syndrome (ACS) is a syndrome due to decreased blood flow in the coronary arteries such that part of the heart muscle is unable to function properly or dies. The most common symptom is chest pain which often radiating to the left shoulder or angle of the jaw, crushing, central and associated with nausea and sweating(1). Acute coronary syndrome is usually caused by one of three problems: ST elevation myocardial infarction (STEMI, 30\%), non ST elevation myocardial infarction (NSTEMI, $25 \%)$, or unstable angina (38\%). (2)

Acute myocardial infarction (AMI) indicates irreversible myocardial injury resulting in necrosis of a significant portion of myocardium (generally $>1 \quad \mathrm{~cm}$ ). Atherosclerosis is the most common cause of myocardial infarction and its major risk factors are hyperlipidemia, diabetes, smoking, hypertension, gender and age. Endothelial dysfunction and inflammation have a major role in the initiation of the atherosclerotic plaque formation. (3). Myocardial infarction occurs most often in the early morning hours. Mechanisms that may explain this circadian variation include morning increase in sympathetic tone and plasma catecholamine leading to increases in blood pressure, heart rate, coronary vascular tone, and myocardial contractility resulting in increased myocardial demand. (4)

Electrocardiography monitoring is recommended as soon as possible in all patients with suspected STEMI in order to detect life threatening arrhythmias and allow prompt defibrillation if indicated. When a STEMI is suspected, a 12-lead ECG must be acquired and interpreted as soon as possible at the time of first medical contact with a target delay of $\leq 10 \mathrm{~min}$ to facilitate early STEMI diagnosis and triage. (5)
The primary objectives of the study were identification of the culprit artery responsible for STEMI from ECG leads, localization of the site of lesion in the artery either proximal, mid or distal part, by using Fiol's algorithm and correlation with coronary angiogram.

\section{Materials and Methods}

Thisis a cross-sectional observational study conducted in Tanta University Emergency Hospital from March 2017 to end of February 2018 on16 patients presented to Emergency medicine department with acute respiratory failure (ARF) and admitted to intensive care unit. The study was approved by the Research Ethics Committee of Tanta University Faculty of Medicine

\section{Study setting and population}

This observational cross-sectional study analyzed 180 cases that came with acute myocardial infarction to cardiology department of Tanta University Hospital in a period from the first of May 2016 up to the end of May 2018.

Each patient was subjected to clinical examination and investigations in the form of 12-lead ECG, Echocardiography, Serum cardiac troponin and CK-MB, and coronary angiography for the possibility of primary percutaneous coronary intervention (PCI).

Typical chest pain lasting more than 30 minutes accompanied by ST-elevation at the J-point in two contiguous leads associated with elevation of cardiac markers were included in this study but we exclude patients with Previous history of myocardial infarction, Previous history of coronary artery bypass graft $(\mathrm{CABG})$ surgery, ECG evidence of left bundle branch block (LBBB), preexcitation, paced rhythm and acute coronary syndrome other than STEMI. 


\section{Study protocol}

All patients presented with ST-segment elevation in precordial leads, which was considered to be a sign of LAD occlusion, were evaluated to predict the placement of occlusion in LAD. Criteria used to locate the occlusion above or below the first diagonal artery (D1) and the first septal artery (S1) are based on deviations of the ST-segment in inferior, anterolateral and/or aVR leads.

All these deviations were evaluated in all patients to predict the site that may be:

Occlusion proximal to D1 (ST depression in leads II, III, aVF)

Occlusion distal to D1 elevation/Isoelectric in leads II, III, aVF)

Occlusion proximal to S1 (ST depression/Isoelectric in leads V5-V6 and/or ST elevation in aVR/V1).

Dominant ST elevation in inferior leads indicates evolving MI of the inferior-posterior zone with possible extension to lateral wall, due to occlusion in the RCA $(70-80 \%$ of cases) or (LCX) (20-30\% of cases). To know more details about which artery was affected and its characteristics we did the following: -

In 12-lead surface ECG we have to, at first check ST segment in lead I. ST depression in lead I indicates that RCA is more probable the culprit artery, while ST elevation in lead I probably indicates that LCX is the affected artery.

In case of isoelectric ST in lead I we must check if ST elevation in lead II is equal or greater than ST elevation in lead III. In this case the affected artery is usually LCX and if it is the contrary (ST elevation is greater in lead III than in lead II), it is more probable that RCA is the culprit artery. There may be some doubts; to be sure we have to proceed to another step.

We checked if ST depression in V1 divided by ST elevation in lead III is greater or lower than 1 . In the first case, LCX is the probable culprit artery while in the second cases (ratio of ST depression in V1 to ST elevation in III is equal or lower than 1) RCA is the culprit artery.

\section{Right ventricular myocardial infarction}

ST-segment elevation in lead V1 in association with ST-segment elevation in inferior leads (with greater elevation in lead III than in lead II) is highly correlated with the presence of RVMI. ST-segment elevation of more than $1 \mathrm{~mm}$ in lead V4 $\mathrm{R}$ with an upright $\mathrm{T}$ wave in that lead is the most sensitive ECG sign of RVMI.

\section{Posterior wall myocardial infarction}

The standard 12-lead ECG is a relatively insensitive tool for detecting posterior wall MI (PWMI). This is due to the absence of standard leads facing the posterior wall of LV. During acute inferior infarction, STsegment elevation in the posterior chest leads V7 through V9 identifies those patients with concomitant posterior wall involvement. It can be caused by lesions in the RCA or LCX which may be indirectly recognized by reciprocal ST depression in precordial leads V1-V3.

Each patient was subjected to electrocardiogram with the use of special leads e.g. Right-sided leads in inferior wall MI and culprit artery was identified using Fiol's algorithm. Next, every patient was subjected to coronary angiogram with or without primary PCI to confirm the electrocardiographic prediction of the infarct- 
related artery. The extent of coronary artery disease and the morphology of all coronary artery lesions were determined.

\section{Outcome measures}

Coronary angiography (with or without PCI) was performed to detect the culprit lesion within 12 hours after admission of the patient. And considered the gold standard for diagnosis of the culprit artery. The sensitivity, specificity, and likelihood ratios and their 95\% confidence intervals were calculated.

\section{Results and Discussion}

Basic clinical data showed that most of MI cases $(64.44 \%)$ were in the age group of 41 60 years, Male patients constituted $73.33 \%$, Chest pain presentation ranged from 1-16 hours with mean \pm SD $5.356 \pm 4.798$, Some of the cases $(22.22 \%)$ were smokers, $17.78 \%$ diabetic, $14.44 \%$ hypertensive, $11.11 \%$ were obese, $6.67 \%$ have positive family history of coronary artery disease, $5.56 \%$ have hyperlipidemia and $22.22 \%$ without any comorbidities.

Among all myocardial infarction patients, ECG shows left anterior descending (LAD) branch affection in $116 / 180$ patients (64.44\%), which was as follow; $13.33 \%$ proximal to $\mathrm{S} 1,6.67 \%$ proximal to $\mathrm{D} 1$ and $44.44 \%$ distal to D1. Right coronary artery (RCA) presented in 56/180 patients $(31.11 \%)$ as follow; $11.11 \%$ proximal to RV branch and $20 \%$ distal to RV branch. Left circumflex artery (LCX) is represented only in 8/180 patients $(4.44 \%)$.

There is statistically significant relation between ECG and complications. Tachyarrhythmia occurred in $(17.24 \%)$ of patients and cardiogenic shock in $(10.34 \%)$, which have higher percentage of complications appeared only in LAD affection in ECG. Bradyarrythmia was found in $(7.14 \%)$ of patients with RCA affection, while bradyarrhythmia associated with right side heart failure appeared in (14.29\%) of patients with RCA affection. There are $(75.56 \%)$ of patients passed without complications.

There is high statistically significant association between ECG and coronary angiography finding, from 116 cases of LAD appeared in ECG, there is only 108 case correlated with coronary angiography result, while other 8 cases were related to RCA. As regard to the 56 cases of RCA appeared in ECG, there is only 36 case correlated with coronary angiography result, 8 cases related to LCX and 12 case related to LAD (distal to D1). As regard to 8 cases of LCX appeared in ECG, there is only 4 cases correlated with coronary angiography result, while other 4 cases were related to RCA (distal to RV).

In this study, as regard to LAD; there is high specificity and positive predictive value of ECG $(100 \%)$ to LAD lesions proximal to S1. LAD lesions proximal to D1 have high specificity $(83.33 \%)$ and negative predictive value $(94.59 \%)$ related to ECG. LAD lesions distal to D1 have high specificity $(88 \%)$ and sensitivity $(75 \%)$ in relations to ECG findings. As regard to RCA; ECG is highly specific $(96.77 \%)$ to RCA lesions proximal to $\mathrm{RV}$ part and lesion distal to RV (93.55\%) while sensitivity to both were low $(21.43 \%$, $42.86 \%$ ) respectively. According to LCX; ECG has high specificity $(95.35 \%)$ and moderate sensitivity (50\%) to LCX lesions.

In this study; According to LAD, there is high specificity of ECG (100\%) to detect LAD lesions proximal to S1 more than distal to D1 (88\%), and proximal to D1 $(83.33 \%)$. The highest sensitivity (75\%) occurred in LAD lesions distal to D1. As regard to RCA; ECG is only sensitive by $42.86 \%$ to detect lesions 
distal to RV branch and highly specific $(96.77 \%)$ to lesions proximal to RV branch more than specificity to distal RV branch (93.55\%). As regard to LCX; ECG has high specificity $95.35 \%$ and moderate sensitivity (50\%).

Chakraborty et al., (2017); showed that, as regarding to LAD lesions, ECG had highest sensitivity (90\%) to detect lesions proximal to S1 part and highest specificity (86\%) to lesions proximal to D1 part of LAD. According to RCA and LCX, ECG had 100\% sensitivity to RCA and $100 \%$ specificity to LCX.(6)

Ghosh et al., (2013); reported that, as regarding to LAD lesions, ECG had (92.86\%) specificity to detect lesions proximal to $\mathrm{S} 1$ then lesions proximal to D1 $(90 \%)$ while sensitivity was low for all parts. According to RCA, there was $100 \%$ sensitivity for lesions distal to RV branch while specificity was slightly higher for lesions proximal to RV branch $(92.86 \%)$ than lesions distal to RV branch $(89.47 \%)$. The specificity was very high for LCX lesions (100\%).

George et al., (2016) used the same electrocardiographic algorithm of Chakraborty et al., (2017) where proximal LAD lesions had specificity (88.5\%), sensitivity (57.8\%) and PPV (87.5\%). As regarding to RCA lesions, it had specificity (92.4\%), sensitivity (63.8\%), PPV (85\%) and NPV (79.03\%). According to Lcx lesions, specificity was high $(89.03 \%)$ while it had very low sensitivity (7.14\%). This study didn't classify coronary arteries from the start as in our study and studies made by Chakraborty et al., (2017) and Ghosh et al., (2013).(6)

Fiol et al., (2009) revealed that, LAD lesions proximal to S1 had high sensitivity $(100 \%)$ and low specificity (41\%), lesions proximal to
D1 had sensitivity (77\%) and specificity (84\%), while lesions distal to D1 had high specificity $(100 \%)$ and low sensitivity (41\%).(7)

The difference between our results and other studies in sensitivity and specificity of the ECG in acute MI was related to individual variations in coronary anatomy as well as by the presence of pre-existing coronary artery disease particularly in patients with a previous MI, collateral circulation, or previous coronary-artery bypass surgery.

It also affected by time of ECG taken, time of presentation of patients, thrombolytic therapy, dominant vessels and presence of multi vessel affection.

There was a total agreement between our study and Ghosh et al., (2013) as regard the higher specificity and low sensitivity for all parts of LAD cases. Also, there was agreement between our study and George et al., (2016) in higher specificity and lower sensitivity only as regarding to LAD lesions proximal to S1. On the other hand, Chakraborty et al., (2017) and Fiol et al., (2009) had high sensitivity to proximal S1 part of LAD.

As regarding to RCA, there was disagreement between our study and Ghosh et al., (2013) in sensitivity of distal RV part which was higher in Ghosh et al., (2013) more than our study. Sensitivity was also very high in Chakraborty et al., (2017) study but very low specificity in all cases. As regarding to LCX, there was a total agreement between all studies; specificity was very high in comparison with its sensitivity, which was very low (Table 1-4).

\section{Limitation of the study}

This study was single center study with small size sample. 
Table.1 Relation between age and sex in study group

\begin{tabular}{|c|c|c|c|c|c|c|c|c|}
\hline \multirow[t]{3}{*}{ Age groups } & \multicolumn{6}{|c|}{ Sex } & \multicolumn{2}{|c|}{ Chi-Square } \\
\hline & \multicolumn{2}{|c|}{ Male } & \multicolumn{2}{|c|}{ Female } & \multicolumn{2}{|c|}{ Total } & & \\
\hline & $\mathbf{N}$ & $\%$ & $\mathbf{N}$ & $\%$ & $\mathbf{N}$ & $\%$ & $\mathbf{X}^{2}$ & P-value \\
\hline 20-40 Years & 16 & 12.12 & 0 & 0.00 & 16 & 8.89 & \multirow[t]{4}{*}{6.959} & \multirow[t]{4}{*}{$0.031 *$} \\
\hline 41-60 Years & 84 & 63.64 & 32 & 66.67 & 116 & 64.44 & & \\
\hline$>60$ Years & 32 & 24.24 & 16 & 33.33 & 48 & 26.67 & & \\
\hline Total & 132 & 100.00 & 48 & 100.00 & 180 & 100.00 & & \\
\hline
\end{tabular}

Table.2 Distribution of ECG changes among studied groups

\begin{tabular}{|c|c|c|}
\hline \multicolumn{2}{|c|}{ ECG } & \% \\
\hline & N & 13.33 \\
\hline LAD proximal to S1 & 24 & 6.67 \\
\hline LAD proximal to D1 & 12 & 44.44 \\
\hline LAD distal to D1 & 80 & 11.11 \\
\hline RCA proximal to RV & 20 & 20.00 \\
\hline RCA distal to RV & 36 & 4.44 \\
\hline LCX & 8 & 100.00 \\
\hline Total & 180 & \\
\hline
\end{tabular}

Table.3 Correlation between ECG and coronary angiography findings

\begin{tabular}{|c|c|c|c|c|c|c|c|c|c|c|}
\hline \multirow{3}{*}{$\begin{array}{c}\text { Coronary } \\
\text { angiography }\end{array}$} & \multicolumn{8}{|c|}{ ECG } & \multirow{2}{*}{\multicolumn{2}{|c|}{ Chi-Square }} \\
\hline & \multicolumn{2}{|c|}{ LAD } & \multicolumn{2}{|c|}{ RCA } & \multicolumn{2}{|c|}{ LCX } & \multicolumn{2}{|c|}{ Total } & & \\
\hline & $\mathbf{N}$ & $\%$ & $\mathbf{N}$ & $\%$ & $\mathbf{N}$ & $\%$ & $\mathbf{N}$ & $\%$ & $\mathrm{X}^{2}$ & P-value \\
\hline Proximal S1 & 16 & 13.79 & 0 & 0.00 & 0 & 0.00 & 16 & 8.89 & 126.004 & $<0.001^{*}$ \\
\hline ProximalD1 & 32 & 27.59 & 0 & 0.00 & 0 & 0.00 & 32 & 17.78 & & \\
\hline Distal D1 & 60 & 51.72 & 12 & 21.43 & 0 & 0.00 & 72 & 40.00 & & \\
\hline Proximal RV & 4 & 3.45 & 12 & 21.43 & 0 & 0.00 & 16 & 8.89 & & \\
\hline Distal RV & 4 & 3.45 & 24 & 42.86 & 4 & 50.00 & 32 & 17.78 & & \\
\hline LCX & 0 & 0.00 & 8 & 14.29 & 4 & 50.00 & 12 & 6.67 & & \\
\hline Total & 116 & 100.00 & 56 & 100.00 & 8 & 100.00 & 180 & 100.00 & & \\
\hline
\end{tabular}

$(\mathrm{LAD}=$ Left anterior descending artery, $\mathrm{RCA}=$ Right coronary artery, LCX= Left circumflex artery, $\mathrm{D}=\mathrm{Diagonal}$, $\mathrm{S}=$ Septal, $\mathrm{RV}=$ right ventricular branch).

Table.4 Sensitivity and Specificity of ECG to coronary angiography

\begin{tabular}{|c|c|c|c|c|c|}
\hline & Sensitivity & Specificity & PPV & NPV & Accuracy \\
\hline LAD Proximal S1 & 66.67 & 100.00 & 100.00 & 95.12 & 95.56 \\
\hline LAD Proximal D1 & 33.33 & 83.33 & 12.50 & 94.59 & 80.00 \\
\hline LAD Distal D1 & 75.00 & 88.00 & 83.33 & 81.48 & 82.22 \\
\hline RCA Proximal RV & 21.43 & 96.77 & 75.00 & 73.17 & 73.33 \\
\hline RCA Distal RV & 42.86 & 93.55 & 75.00 & 78.38 & 77.78 \\
\hline LCX & 50.00 & 95.35 & 33.33 & 97.62 & 93.33 \\
\hline
\end{tabular}


Although coronary angiography is the gold standard for determining the infarct-related artery in acute myocardial infarction, the present study demonstrates that ECG is an easily and widely available inexpensive tool which help in the routine clinical practice to categorize and optimize STEMI patients by detection of artery occluded into either right coronary artery or left anterior descending coronary artery or left circumflex coronary artery as the infarcted related culprit vessel and predict the possible complication

\section{Authorship}

Mohamed Shama was behind the idea of the research, collected the data of the patients and follow up the patients in the Intensive Care Unit. Ghada A. Attia and Atef H. Teima did the statistics. Abdelhady Taha wrote the manuscript. All authors revised the manuscript.

\section{Acknowledgments}

Prof. Mohamed Elhenaidy head of emergency medicine department.

\section{Ethical approval}

Ethical approval for the study was obtained from ethical committee of Tanta University Faculty of Medicine before the start of the research.

\section{Human rights}

The study protocol conforms to the ethical guidelines of the 1975 Declaration of Helsinki as reflected in a priori approval by the institution's human research committee.

\section{References}

Kumar A, Cannon CP, editors. Acute coronary syndromes: diagnosis and management, part I. Mayo Clinic Proceedings; 2009: Elsevier.

Jneid H, Anderson JL, Wright RS, Adams CD, Bridges CR, Casey DE, et al., 2012 ACCF/AHA focused update of the guideline for the management of patients with unstable angina/non-STelevation myocardial infarction (updating the 2007 guideline and replacing the 2011 focused update): a report of the American College of Cardiology Foundation/American Heart Association Task Force on Practice Guidelines. Journal of the American College of Cardiology. 2012;60(7):64581.

Thygesen K, Alpert JS, Jaffe AS, Simoons ML, Chaitman BR, White HD. Third universal definition of myocardial infarction. Circulation. 2012; 126(16): 2020-35.

White WB. Cardiovascular risk and therapeutic intervention for the early morning surge in blood pressure and heart rate. Blood pressure monitoring. 2001; 6(2): 63-72.

Members ATF, Steg PG, James SK, Atar D, Badano LP, Lundqvist CB, et al., ESC Guidelines for the management of acute myocardial infarction in patients presenting with ST-segment elevation: The Task Force on the management of ST-segment elevation acute myocardial infarction of the European Society of Cardiology (ESC). European heart journal. 2012; 33(20): 2569-619.

Chakraborty S, Majumder B, Sarkar D, Chatterjee S. A Simple Non-invasive ECG Technique to Localize Culprit Vessel Occlusion Site in ST-Elevation Myocardial Infarction (STEMI) Patients. J Clin Exp Cardiolog. 2017; 8(556): 2.

Fiol M, Carrillo A, Cygankiewicz I, Velasco J, Riera M, Bayés- Genis A, et al., A new electrocardiographic algorithm to 
locate the occlusion in left anterior descending coronary artery. Clinical Cardiology: An International Indexed and Peer- Reviewed Journal for
Advances in the Treatment of Cardiovascular Disease. 2009; 32(11): E1-E6.

\section{How to cite this article:}

Mohamed Abdelhady Shama, Mohamed Naseem Hussaien, Mona Adel ELsaiedy and Raghda Ghonimy Elsheikh. 2019. Usefulness of the Electrocardiogram as a Tool to Localize Culprit Vessel Occlusion Site in Acute ST - Segment Elevation Myocardial Infarction. Int.J.Curr.Microbiol.App.Sci. $\quad$ 8(06): 1988-1995. https://doi.org/10.20546/ijcmas.2019.806.237 